\title{
Familienpolitik auf dem Prüfstand
}

\section{Corinne Knöpfel}

\section{Relevanz}

Zwanzig Tage Vaterschaftsurlaub fordert eine Volksinitiative. Die Initianten sind überzeugt, dass ein solcher den jungen Familien nützt und längst überfällig ist. Der Bundesrat hingegen fürchtet um die Wettbewerbsfähigkeit der Schweiz und lehnt die Initiative ab. Was sind die Folgen des Vaterschaftsurlaubs wirklich? Kann er helfen, Beruf und Familie besser in Einklang zu bringen? Wird er die Erwerbsbeteiligung der Frauen steigern und ihnen helfen, rascher an den Arbeitsplatz zurückzukehren? Wie wirkt er sich auf ihre Löhne aus? Sind gar positive Folgen für die Geburtenhäufigkeit zu erwarten? Schliesslich wäre wichtig zu wissen, wie der Vaterschaftsurlaub im Vergleich zu anderen Instrumenten der Familienpolitik abschneidet.

Christian Keuschnigg und Michael Kogler

\section{Quelle}

Der nachfolgende Text ist eine Zusammenfassung von: Olivetti, Claudia and Barbara Petrongolo (2017), The Economic Consequences of Family Policies: Lessons from a Century of Legislation in High-Income Countries, Journal of Economic Perspectives 31, 205-230.

Regelungen für Frauen und Familien im Arbeitsumfeld haben in Europa eine lange Tradition. Bereits während der Industrialisierung wurden im Rahmen des

C. Knöpfel $(\bowtie)$

Universität St. Gallen, St. Gallen, Schweiz

E-Mail: corinne.knoepfel@student.unisg.ch

(C) Der/die Autor(en) 2018

C. Keuschnigg (Hrsg.), Inklusives Wachstum und wirtschaftliche Sicherheit, https://doi.org/10.1007/978-3-658-21344-2_10 
Arbeitnehmerschutzes spezifische Vorschriften für Frauen eingeführt. Anfang des 20. Jahrhunderts ergänzten einige Länder diese um einen unbezahlten Mutterschaftsurlaub. Dadurch erhielten Mütter die Garantie, wieder an ihren bisherigen Arbeitsplatz zurückkehren zu können. Später wurden finanzielle Unterstützungen während des Mutterschaftsurlaubs geschaffen. Schweden führte in den 1970er Jahren als erstes Land einen sechsmonatigen Elternurlaub ein, welcher von Müttern und Vätern gleichermassen beansprucht werden konnte. Auch die Europäische Union veröffentlichte in den 1990er Jahren eine Richtlinie für einen dreimonatigen Elternurlaub und stellte die Vereinbarkeit von Arbeit und Familie in den Vordergrund. Was sind die Folgen für Familien, Wirtschaft und Gesellschaft? Claudia Olivetti vom Boston College und Barbara Petrongolo von der Londoner Queen Mary University sind diesen Fragen auf den Grund gegangen. Sie stützten sich in ihrer Untersuchung sowohl auf eigene Schätzungen als auch auf eine Vielzahl existierender Studien. Heute sehen alle reichen, industrialisierten Länder zumindest einen bezahlten Mutterschaftsurlaub vor. Ausnahme sind die USA, wo dieser unbezahlt bleibt. Neben den Elternurlaub sind nach und nach weitere familienfördernde Massnahmen getreten, wie die öffentliche Finanzierung von Krippenplätzen, Teilzeitarbeit, oder flexible Arbeitszeiten. Allerdings streiten sich Befürworter und Gegner nach wie vor über Nutzen und Kosten dieser Politik. Den Fortschritten in der Gleichberechtigung, der Förderung der Kindesentwicklung und der besseren Vereinbarkeit von Beruf und Familie stehen hohe Kosten und verpasste Berufserfahrung - verbunden mit beeinträchtigten Karrierechancen - gegenüber.

Wie beeinflusst die Familienpolitik die Erwerbstätigkeit und die Löhne von Frauen? Die Wissenschaftlerinnen starten mit den Ergebnissen einer früheren Untersuchung für neun EU Länder (1969-93), wonach eine bezahlte Elternzeit von bis zu drei Monaten die Beschäftigungsquote von Frauen um 3 bis $4 \%$ erhöht, aber keinen Effekt auf die Gehälter hat. Eine Verlängerung des Elternurlaubs, z. B. auf neun Monate, kann jedoch die Erwerbsquote von Frauen nicht mehr steigern, während sich ihre Löhne um $3 \%$ verringern. Dieses Resultat wurde seither von zahlreichen weiteren Studien bestätigt. Zudem erwähnen sie eine Studie für OECD Länder (1990-2010) mit dem Ergebnis, dass familienpolitische Massnahmen geschlechtsspezifische Unterschiede bei Beschäftigung und Gehältern verringern. Allerdings ist die empirische Evidenz nicht durchgehend positiv. Eine Untersuchung zur Einführung des Mutterschaftsurlaubs in den USA konnte keinen Einfluss auf Erwerbsquote und Löhne von Frauen feststellen. Weitere Studien aus den USA fanden sogar, dass Mutterschaftsurlaub und staatliche 
Unterstützung zu einer tieferen Beschäftigung von Frauen führt. Dagegen zeitigen öffentliche Ausgaben im Bereich der Kinderbetreuung positive Effekte.

Eine bezahlte Elternzeit von drei Monaten erhöht die Beschäftigungsquote von Frauen um 3 bis $4 \%$, ohne die Löhne zu beeinträchtigen. Ein längerer Elternurlaub erhöht die Beschäftigung nicht weiter, senkt aber die Löhne von Frauen um 3 \%.

Olivetti und Petrongolo schätzen selber den Effekt der Familienpolitik auf Beschäftigung und Einkommen von Frauen sowie auf die Geburtenrate in 30 OECD Ländern. Sie konzentrieren sich auf Massnahmen wie z. B. Mutter- und Vaterschaftsurlaub oder Ausgaben für frühkindliche Betreuung. Dazu vergleichen die Forscherinnen aggregierte Daten zu Arbeitsmarkt und Fertilität in den betroffenen Ländern.

Abb. 1 zeigt die Entwicklung der Beschäftigungsquote von Frauen zwischen 1970 und 2010. Die Quote von berufstätigen Frauen ist in fast allen Ländern

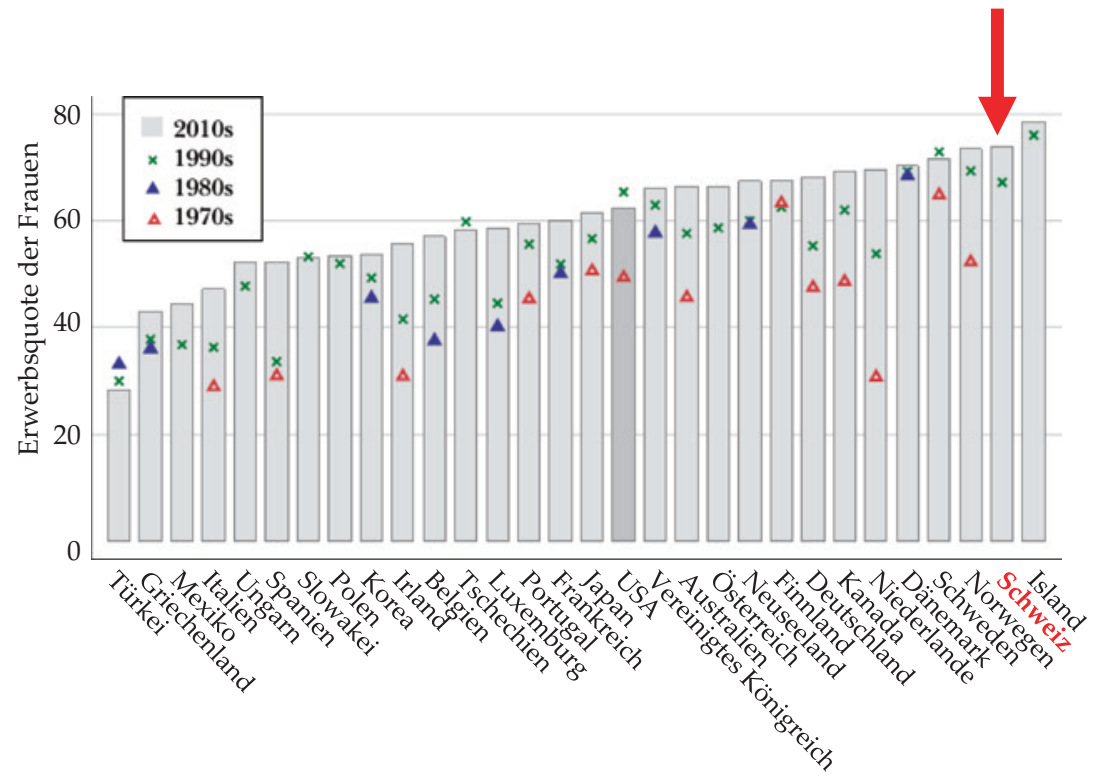

Abb. 1 Beschäftigungsquote von Frauen im Alter von 25 bis 54 Jahren von 1970 bis 2010. Als beschäftigt gelten Frauen, die mindestens eine Stunde pro Woche arbeiten. (Quelle: Olivetti \& Petrongolo, 2017, 215) 
gestiegen. Heute sind durchschnittlich $60 \%$ aller Frauen erwerbstätig, verglichen mit $49 \%$ in den 1980er Jahren. Die Daten deuten auch darauf hin, dass bezahlter und unbezahlter Mutterschaftsurlaub mit geringeren Unterschieden in der Beschäftigungsquote von Männern und Frauen einhergeht. Der Vaterschaftsurlaub hingegen steht in keiner Verbindung zu Lohn oder Beschäftigung. Öffentliche Ausgaben für Kinderbetreuung sowie flexible Arbeitsmöglichkeiten wiederum gehen mit einer höheren Beschäftigungsquote für Frauen und einer geringeren Geschlechterungleichheit in der Erwerbsquote einher. Ein besseres Angebot an Kinderbetreuung ist ausserdem mit einer höheren Geburtenrate verbunden.

Die Schätzungen zeigen, dass ein Mutterschaftsurlaub von bis zu fünfzig Wochen die Quote erwerbstätiger Frauen erhöht. Die Effekte sind allerdings gering. Würden die USA den unbezahlten Mutterschaftsurlaub von derzeit 12 auf 70 Wochen erhöhen, wie dies z. B. in Schweden üblich ist, so stiege die Erwerbsquote für Frauen um 1,4 Prozentpunkte. Dies ist im Vergleich zur momentanen Frauenerwerbsquote in den USA von $62 \%$ wenig. Bei längerer Urlaubszeit wird der Beschäftigungseffekt schwächer oder sogar negativ.

Zudem deutet die Analyse auf unterschiedliche Effekte der Familienpolitik abhängig von Qualifikation und Ausbildung der Frauen hin. Vor allem geringqualifizierte Frauen profitieren von einem Mutterschaftsurlaub und werden dadurch häufiger erwerbstätig. Die Erwerbsquote von hochqualifizierten Frauen nimmt hingegen kaum zu, während ihre Löhne tendenziell sinken. Ein Berufsunterbruch ist für eine hoch qualifizierte Beschäftigung eher ungünstig.

Eine wichtige familienpolitische Massnahme ist der Ausbau der frühkindlichen Betreuung und Bildung. In den betrachteten OECD-Ländern lagen 2014 die Ausgaben dafür zwischen 0,4\% (USA) und $2 \%$ des BIP. Ein Anstieg dieser Ausgaben um einen Prozentpunkt resultiert in einer um 3,6 Prozentpunkte höheren Beschäftigungsquote. Zudem erhöht dieselbe Ausgabensteigerung im Durchschnitt die Geburtenrate um bis zu 0,2 zusätzliche Kinder pro Frau.

\begin{abstract}
Eine Verlängerung des Mutterschaftsurlaubs von 12 auf 70 Wochen steigert die Frauenerwerbsquote um 1,4 Prozentpunkte. Die Erhöhung der Ausgaben für Kinderbetreuung um $1 \%$ des BIP führt zu einem Anstieg der Frauenerwerbsquote von 3,6 Prozentpunkten.
\end{abstract}

Wie reagieren die einzelnen Haushalte, Arbeitnehmer und Arbeitgeber auf familienpolitische Massnahmen? Die Forscherinnen stützen sich dabei auf mehrere 
Studien, welche familienpolitische Reformen mithilfe individueller Daten analysieren. Demnach hatte die Verlängerung der Elternzeit in Österreich im Jahr 1990 von einem auf zwei Jahre einen signifikanten Effekt auf die Fertilität. Die Geburtenrate stieg um 12 Kinder pro 100 Frauen. Gleichzeitig ging die Erwerbsquote von Frauen zumindest in den ersten drei Jahren nach der Geburt zurück. Weitere Analysen zeigen, dass eine grosszügige finanzielle Unterstützung die Rückkehr an den Arbeitsplatz ebenfalls verzögert. In Deutschland oder Norwegen etwa führte die Verlängerung der Elternzeit dazu, dass Frauen nach der Geburt länger zu Hause blieben und später an ihren Arbeitsplatz zurückkehrten. Langfristig hatte dies jedoch kaum Folgen für die Erwerbstätigkeit oder die Einkommen.

Nur wenige Studien untersuchen die Auswirkungen eines Vaterschaftsurlaubs, denn das Phänomen ist vergleichsweise jung. Schweden führte 1995 einen einmonatigen Vaterschaftsurlaub ein. Die Männer nutzten zwar vermehrt diese Möglichkeit nach der Geburt ihres Kindes. Ihr Anteil an der Kinderbetreuung hat sich dadurch aber nicht erhöht.

Der Vaterschaftsurlaub in Schweden führte dazu, dass sich Männer nach der Geburt ihres Kindes zwar vermehrt freigenommen haben. Der Anteil der Väter an der Kinderbetreuung hat sich jedoch nicht erhöht.

Subventionen für die Kinderbetreuung sind schliesslich ein weiterer Ansatz, Familien zu unterstützen. In den USA zeigte sich, dass staatliche Programme für vierjährige Kinder einen geringen positiven Effekt auf die Erwerbstätigkeit wenig qualifizierter Arbeitnehmerinnen haben. Ähnliche Untersuchungen fanden positive Effekte von Kindergärten auf die Erwerbstätigkeit alleinstehender Mütter. In Kanada schätzten Wissenschaftler einen stark positiven Effekt von Kinderbetreuungszulagen für Vierjährige. Die Erwerbstätigkeit von Müttern stieg um 8 Prozentpunkte und der Effekt blieb auch langfristig stabil.

Was können wir aus 100 Jahren Familienpolitik in der industrialisierten Welt lernen? Wie immer ist die Antwort nicht ganz eindeutig. Länderübergreifende Studien finden für eine moderate Elternzeit positive Effekte auf die Erwerbsquote von Frauen, die sich ins Negative wenden, wenn die Elternzeit zu grosszügig angelegt wird. Die Vorteile im Hinblick auf Erwerbstätigkeit und Lohn sind vor allem für gering qualifizierte Arbeitnehmerinnen stärker ausgeprägt. Untersuchungen von 
länderspezifischen Reformen zeigen, dass Elternzeit die Rückkehr an den Arbeitsplatz verzögert, aber die Erwerbsquote langfristig wenig bis gar nicht beeinflusst. Durchwegs positive Effekte zeigen hingegen Ausgaben für die Kinderbetreuung. Solche Investitionen bauen Ungleichheiten zwischen Männern und Frauen auf dem Arbeitsmarkt ab und regen zudem die Geburtenraten an.

Open Access Dieses Kapitel wird unter der Creative Commons Namensnennung 4.0 International Lizenz (http://creativecommons.org/licenses/by/4.0/deed.de) veröffentlicht, welche die Nutzung, Vervielfältigung, Bearbeitung, Verbreitung und Wiedergabe in jeglichem Medium und Format erlaubt, sofern Sie den/die ursprünglichen Autor(en) und die Quelle ordnungsgemäß nennen, einen Link zur Creative Commons Lizenz beifügen und angeben, ob Änderungen vorgenommen wurden.

Die in diesem Kapitel enthaltenen Bilder und sonstiges Drittmaterial unterliegen ebenfalls der genannten Creative Commons Lizenz, sofern sich aus der Abbildungslegende nichts anderes ergibt. Sofern das betreffende Material nicht unter der genannten Creative Commons Lizenz steht und die betreffende Handlung nicht nach gesetzlichen Vorschriften erlaubt ist, ist für die oben aufgeführten Weiterverwendungen des Materials die Einwilligung des jeweiligen Rechteinhabers einzuholen.



